\title{
ECHINODORUS AUSTRALIS (ALISMATACEAE), UN NUEVO SINÓNIMO PARA HELANTHIUM BOLIVIANUM
}

\author{
Echinodorus australis (Alismataceae), a new synonym for Helanthium bolivianum
}

CHRISTIAN A. ZANOTTI' 1 (D) \& ADELA M. PANIZZA² (D)

\begin{abstract}
Resumen: Echinodorus australis Rataj, una especie del sur de Brasil y el norte de Argentina, es aquí sinonimizada bajo Helanthium bolivianum (Rusby) Lehtonen \& Myllys.
\end{abstract}

Palabras clave: Alismataceae, Argentina, Brasil, sinonimia, taxonomía.

Summary: Echinodorus australis Rataj, a species from southern Brazil and northern Argentina, is here synonymized under Helanthium bolivianum (Rusby) Lehtonen \& Myllys.

Key words: Alismataceae, Argentina, Brazil, synonymy, taxonomy.

\section{Introducción}

La familia Alismataceae presenta una distribución casi cosmopolita en regiones tropicales a subtropicales, cuenta con 14 géneros y aproximadamente 95 especies de plantas acuáticas o semi-acuáticas (Lehtonen, 2018). Es una familia de importancia económica, ya que algunas de sus especies son utilizadas como plantas ornamentales en acuarios y jardines acuáticos (Kasselmann, 2003; Lehtonen \& Rodríguez-Arévalo, 2005).

El género Helanthium (Benth. \& Hook. f.) Engelm. ex J. G. Sm., de ambientes tropicales y subtropicales de América, ha sido tradicionalmente considerado como subgénero de Echinodorus Rich.(Rataj, 1975, 2004; Haynes \& Holm-Nielsen, 1994; Lot \& Novelo, 1994); no obstante, estudios filogenéticos revelaron que se trata de dos géneros diferentes y que no se encuentran estrechamente relacionados (Lehtonen \& Myllys, 2008). En la actualidad, se reconocen 3 especies dentro de Helanthium: $H$. bolivianum (Rusby) Lehtonen \& Myllys y $H$. tenellum (Mart. ex Schult. \& Schult. f.) Britton, que habitan desde el centro y norte de los EE. UU. hasta el norte de la Argentina (Haynes \& Holm-Nielsen, 1994) y H. zombiense (Jérémie) Lehtonen \& Myllys, endémica de las Islas de Guadalupe, en las Antillas (Lehtonen \& Myllys, 2008). Sin embargo, los límites taxonómicos entre las especies son controversiales debido al alto grado de variabilidad morfológica, lo cual ha sido reflejado en la disparidad en el número de especies, ya sea por diferentes criterios taxonómicos o por la descripción de nuevas entidades (Fassett, 1955; Rataj, 1975, 2004; Haynes \& Holm-Nielsen, 1994; Lot \& Novelo, 1994; Jérémie et al., 2001). Cabe destacar, que las relaciones filogenéticas entre estas tres especies aún no se hallan resueltas y Helanthium podría estar conformado por más especies (Lehtonen \& Myllys, 2008).

En la última revisión sobre el género Echinodorus sensu lato, Rataj (2004: 16) publica E. australis Rataj (incluída en el subgénero Helianthium (Benth. \& Hook. f.) Fassett), nueva especie para el sur de Brasil y norte de Argentina, considerada en la actualidad como una especie

${ }^{1}$ ILPLA (Instituto de Limnología “Dr. Raúl A. Ringuelet”) CONICET-CCT-La Plata / UNLP. Bv. 120 y 62, La Plata (1900), Buenos Aires, Argentina. E-mail: czanotti1979@gmail.com

${ }^{2}$ FacFor- UNaM (Facultad de Cs. Forestales - Universidad Nacional de Misiones). Bertoni 124, Eldorado (3380), Misiones, Argentina. E-mail: adela.maria.panizza@gmail.com 
válida para el Cono Sur (Haynes, 2008). Sin embargo, la descripción original, el material tipo y los materiales adicionales de E. australis se corresponden con $H$. bolivianum, por lo que en esta contribución se considera un nuevo sinónimo.

Helanthium bolivianum (Rusby) Lehtonen \& Myllys, Cladistics 24(2): 228. 2008.

Alisma boliviana Rusby, Mem. New York Bot. Gard. 7: 208. 1927 = Echinodorus bolivianus (Rusby) Holm-Niels., Brittonia 31(2): 276. 1979.

Typus: Bolivia. Reyes, 25 Oct 1921, O. E. White 1540 (lectotypus, NY00214634! designado por Haynes \& Holm-Nielsen, Fl. Neotrop. 64: 14. 1994; isolectotypi, GH00026755!, K000061663!, NY00214633!). Imagen del lectotipo disponible en https://plants.jstor.org/stable/10.5555/al.ap. specimen.ny00214634

Echinodorus austroamericanus Rataj, Darwiniana 16: 18. 1970. Typus: Paraguay. Dep. Central, Ypacaray, "in regione lacus Ypacaray", May 1913, E. Hassler 12654 (holotypus, Z000075267!, isotypi, C, E00319728!, F0045062F!, G00004541!, G00004542!, GOET, GH, K!, NY311442!, S-R-1818!, UC930396!, US00086699!). Imagen del holotipo disponible en la página del herbario $\mathrm{Z}$ : https://www. herbarien.uzh.ch/en/belegsuche.html

Echinodorus australis Rataj, Aqua 1: 16. 2004. Typus: Brasil. Santa Catarina: Mun. Agua Doce, by small river, Campos de Palmas, alt. 1000-1200 m, 3 Dic 1964, L. B. Smith \& R. M. Klein 13449 (holotypus, US01327721!). Syn. nov. (Fig. 1).

La descripción original de E. australis realizada por Rataj (2004) es muy breve y sólo se limita a brindar medidas de las estructuras de la planta. Generalmente, las medidas coinciden con el holotipo, excepto las hojas, que presentan un rango de 20-25 $\times 1,5-2,5 \mathrm{~cm}$ en la descripción original y que en el holotipo van del rango de $2-4 \times$ 0,4-0,5 cm. Aparte de esta discrepancia, las demás medidas observadas en la descripción original y en el holotipo, se ajustan a la de Helianthium bolivianum, argumentado además por la presencia de las marcas pelúcidas lineares en las láminas que Rataj describe e ilustra en el protólogo.

Cabe destacar que los ejemplares citados por Rataj en el protólogo de E. australis (2004: 16), excepto el holotipo (Smith \& Klein 13449) y el único ejemplar citado para la Argentina (Burkart 19669, US01246885!), habían sido considerados previamente por el mismo Rataj (1970: 20), como $E$. austroamericanus. Además, el ejemplar Smith \& Klein 13449, se hallaba citado entre los materiales adicionales de $H$. tenellum por Haynes \& Holm-Nielsen (1994: 13).

Por último, cabe aclarar, que los límites morfológicos entre las dos especies de Helanthium citadas para la Argentina, $H$. bolivianum y H. tenellum (Haynes \& HolmNielsen, 1994; Haynes, 2008), son muy difíciles de discernir, y por esta razón, $H$. tenellum ha sido considerado como un sinónimo de $H$. bolivianum por Jérémie et al. (2001). No obstante, en esta contribución, se sigue el criterio de Lehtonen \& Myllys (2008) quienes consideran tratar a estos dos taxones como entidades separadas, soportados en análisis filogenéticos. Estas dos especies presentan una alta afinidad morfológica, $H$. bolivianum se puede diferenciar de $H$. tenellum por presentar flores de 1-1,5 cm de diám. vs. 0,6-0,8 cm de diám. y por poseer marcas pelúcidas lineares en las láminas vs. ausencia de las mismas (Haynes \& Holm-Nielsen, 1994; Lehtonen, 2018). Por otro lado, los ejemplares de $H$. bolivianum generalmente presentan las hojas lanceolado-elípticas y $H$. tenellum las presenta lineares (Lehtonen, 2018).

\section{Agradecimientos}

A S. Lehtonen por el envío de la publicación original de E. australis y a A. I. Linn (US) por el envío del material original en forma digital. A los dos revisores anónimos que ayudaron a mejorar el manuscrito. 


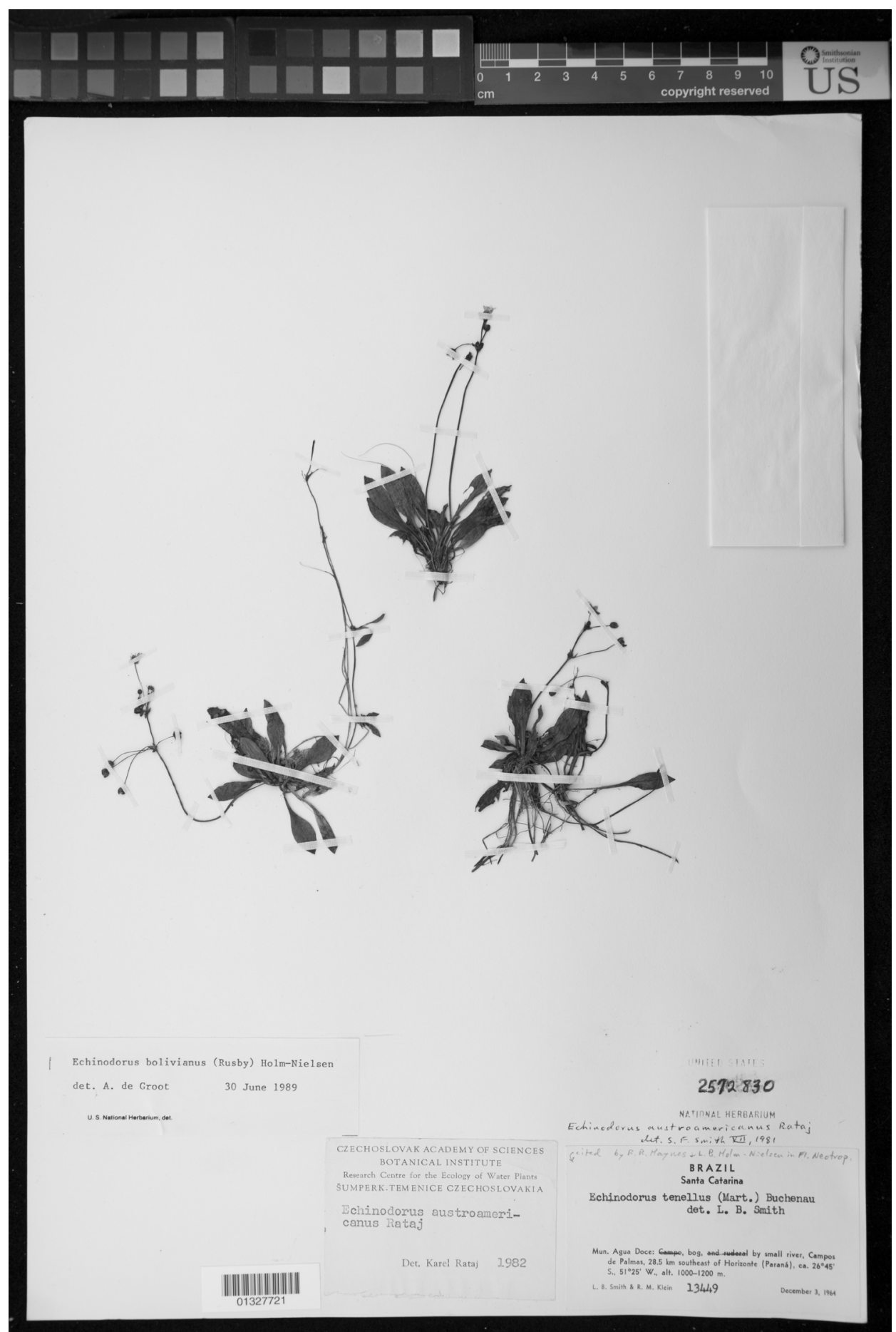

Fig. 1. Holotypus de Echinodorus australis Rataj, L.B. Smith \& R.M. Klein 13449 (US01327721!)

Fig. 1. Holotypus of Echinodorus australis Rataj, L.B. Smith \& R.M. Klein 13449 (US01327721!) 


\section{Bibliografía}

FASSETT, N. C. 1955. Echinodorus in the American tropics. Rhodora 57(677): 133-156. https://doi.org/10.1525/aa.1955.57.1.02a00180

HAYNES, R. R. \& L. B. HOLM-NIELSEN. 1994. The Alismataceae. Flora Neotropica. Monograph 64: 1-112.

HAYNES, R. R. 2008. Alismataceae. En ZULOAGA, F. O., O. MORRONE \& M. J. BELGRANO (eds.), Catálogo de las plantas vasculares del Cono Sur. Monographs in Systematic Botany from the Missouri Botanical Garden 107(1): 169-175.

JÉRÉMIE, J., D. LOBREAU-CALLEN, H. COUDERC \& A. JOSSANG, A. 2001. Une nouvelle espéce d' Echinodorus (Alismataceae) de Guadeloupe (Petites Antilles). Observations palynologiques, cytogénétiques et chimiques. Adansonia 23: 191-203.

KASSELMANN, C. 2003. Aquarium Plants. Krieger Publishing Co., Malabar, FL. 518 pp.
LEHTONEN, S. \& L. A. RODRÍGUEZ ARÉVALO. 2005. Notes on aquarium plant production in Peruvian Amazonia. Ethnobotany Research and Applications 3: 209-214. https://doi.org/10.17348/era.3.0.209-214

LEHTONEN, S. \& L. MYLLYS. 2008. Cladistic analysis of Echinodorus (Alismataceae): simultaneous analysis of molecular and morphological data. Cladistics 24: 218-239. https://doi.org/10.1111/j.1096-0031.2007.00177.x

LEHTONEN, S. 2018. Alismataceae. Flora Paraguay 49: $1-42$.

LOT, A. H. \& A. R. NOVELO. 1994. Alismataceae. En DAVIDSE, G., M. S. SOUSA \& A. O. CHATER (eds.), Flora Mesoamericana 6: Alismataceae a Cyperaceae 6: 3-8.

RATAJ, K. 1975. Revizion [sic!] of the genus Echinodorus Rich. Studie Csav 2: 1-156.

RATAJ, K. 2004. A new revision of the swordplant genus Echinodorus Richard, 1848 (Alismataceae). Aqua, Journal of Ichtyology and Aquatic Biology, Special Publication 1: 1-142. 